\title{
Prenatal molecular testing for Beckwith-Wiedemann and Silver-Russell syndromes: a challenge for molecular analysis and genetic counseling
}

\author{
Thomas Eggermann ${ }^{\star, 1,26}$, Frédéric Brioude ${ }^{2,3,4,26}$, Silvia Russo ${ }^{5,26}$, Maria P Lombardi ${ }^{6,26}$, Jet Bliek ${ }^{6}$, \\ Eamonn R Maher ${ }^{7}$, Lidia Larizza ${ }^{5}$, Dirk Prawitt ${ }^{8}$, Irène Netchine ${ }^{2,3,4}$, Marie Gonzales ${ }^{9,10}$, Karen Grønskov ${ }^{11}$, \\ Zeynep Tümer ${ }^{11}$, David Monk ${ }^{12}$, Marcel Mannens ${ }^{6}$, Krystyna Chrzanowska ${ }^{13}$, Malgorzata K Walasek ${ }^{13}$, \\ Matthias Begemann ${ }^{1}$, Lukas Soellner ${ }^{1}$, Katja Eggermann ${ }^{1}$, Jair Tenorio ${ }^{14,15}$, Julián Nevado ${ }^{14,15}$, \\ Gudrun E Moore $^{16}$, Deborah JG Mackay ${ }^{17}$, Karen Temple ${ }^{17}$, Gabriele Gillessen-Kaesbach ${ }^{18}$, Tsutomu Ogata ${ }^{19}$, \\ Rosanna Weksberg ${ }^{20,21,22,23,24}$, Elizabeth Algar $^{25}$ and Pablo Lapunzina ${ }^{14,15}$
}

Beckwith-Wiedemann and Silver-Russell syndromes (BWS/SRS) are two imprinting disorders (IDs) associated with disturbances of the $11 \mathrm{p} 15.5$ chromosomal region. In BWS, epimutations and genomic alterations within $11 \mathrm{p} 15.5$ are observed in $>70 \%$ of patients, whereas in SRS they are observed in about $60 \%$ of the cases. In addition, $10 \%$ of the SRS patients carry a maternal uniparental disomy of chromosome $711 \mathrm{p} 15.5$. There is an increasing demand for prenatal testing of these disorders owing to family history, indicative prenatal ultrasound findings or aberrations involving chromosomes 7 and 11 . The complex molecular findings underlying these disorders are a challenge not only for laboratories offering these tests but also for geneticists counseling affected families. The scope of counseling must consider the range of detectable disturbances and their origin, the lack of precise quantitative knowledge concerning the inheritance and recurrence risks for the epigenetic abnormalities, which are hallmarks of these developmental disorders. In this paper, experts in the field of BWS and SRS, including members of the European network of congenital IDs (EUCID.net; www.imprinting-disorders.eu), put together their experience and work in the field of $11 \mathrm{p} 15.5$-associated IDs with a focus on prenatal testing. Altogether, prenatal tests of 160 fetuses (122 referred for BWS, 38 for SRS testing) from 5 centers were analyzed and reviewed. We summarize the current knowledge on BWS and SRS with respect to diagnostic testing, the consequences for prenatal genetic testing and counseling and our cumulative experience in dealing with these disorders.

European Journal of Human Genetics (2016) 24, 784-793; doi:10.1038/ejhg.2015.224; published online 28 October 2015

\section{INTRODUCTION}

Disturbances of the chromosomal region 11p15.5 are associated with Beckwith-Wiedemann (BWS, MIM130650) and Silver-Russell syndromes (SRS, MIM180860) (Table 1). Both congenital disorders belong to the group of imprinting disorders (IDs), that is, entities that are characterized by molecular alterations at the imprinted loci. These specifically regulated genes are expressed either only from the maternal or from the paternal gene copy but not biparentally (for a review, see Reik and Walter ${ }^{1}$ ). Many of the currently known 100 imprinted genes are found in clusters, that is, the imprinted loci often comprise multiple genes under coordinated control. This control is mediated by differentially methylated regions (DMRs), stretches of CpG islands in which the cytosine residues are methylated in a parentof-origin manner. So far, nine IDs have been defined, and they are associated with changes in seven imprinting clusters (for a review, see Mackay et $a l^{2}$ and Eggermann $e t a l^{3}$ ).

Among the imprinted loci, the chromosomal region $11 \mathrm{p} 15.5$ has a key role ${ }^{4,5}$ in the etiology of BWS and SRS. It harbors two separate

\footnotetext{
${ }^{1}$ Institut für Humangenetik, RWTH University Aachen, Aachen, Germany; ${ }^{2}$ INSERM, UMR_S 938, Paris, France; ${ }^{3}$ Sorbonne Universities, UPMC Univ Paris 06, Paris, France; ${ }^{4}$ Armand Trousseau Hospital, Pediatric Endocrinology, Paris, France; ${ }^{5}$ Laboratory of Cytogenetics and Molecular Genetics Istituto Auxologico Italiano IRCCS, Milano, Italy; ${ }^{6}$ Department of Clinical Genetics, Academic Center, University of Amsterdam, Amsterdam, The Netherlands; ${ }^{7}$ Department of Medical Genetics, University of Cambridge and NIHR Cambridge Biomedical Research Centre, Cambridge, UK; ${ }^{8}$ Center for Pediatrics and Adolescent Medicine, University Medical Center, Mainz, Germany; ${ }^{9}$ Department of Medical Genetics, Armand Trousseau Hospital, AP-HP, Paris, France; ${ }^{10}$ Sorbonne Universitie, UPMC Univ Paris 06, Paris, France; ${ }^{11}$ Clinical Genetic Unit, Kennedy Center, Rigshospitalet, Copenhagen University Hospital, Glostrup, Denmark; ${ }^{12}$ Imprinting and Cancer Group, Cancer Epigenetic and Biology Program (PEBC), Institut d'Investigació Biomedica de Bellvitge (IDIBELL), Barcelona, Spain; ${ }^{13}$ Department of Medical Genetics, The Children's Memorial Health Insitute, Warsaw, Poland; ${ }^{14}$ Instituto de Genética Médica y Molecular (INGEMM)-IdiPAZ, Hospital Universitario La Paz, Madrid, Spain; ${ }^{15}$ CIBERER, Centro de Investigación Biomédica en Red de Enfermedades Raras, ISCIII, Madrid, Spain; ${ }^{16}$ Fetal Growth and Developmental group, Genetics and Genomic Medicine Programme, UCL-ICH, London, UK; ${ }^{17}$ Human Genetics and Genomic Medicine, Faculty of Medicine University of Southampto; Wessex Clinical Genetics Service, Princess Anne Hospital, Southampton, UK; ${ }^{18}$ Institut für Humangenetik, Universität zu Lübeck, Lübeck, Germany; ${ }^{19}$ Department of Pediatrics, Hamamatsu University School of Medicine, Hamamastu, Japan; ${ }^{20}$ Program in Genetics and Genome Biology, The Hospital for Sick Children, Toronto, Ontario, Canada; ${ }^{21}$ Division of Clinical and Metabolic Genetics, The Hospital for Sick Children, Toronto, Ontario, Canada; ${ }^{22}$ Department of Molecular Genetics, University of Toronto, Toronto, Ontario, Canada; ${ }^{23}$ Institute of Medical Science, University of Toronto, Toronto, Ontario, Canada; ${ }^{24}$ Department of Pediatrics, University of Toronto, Toronto, Ontario, Canada; ${ }^{25}$ Genetics and Molecular Pathology Laboratory, Monash Health and Hudson Institute, Clayton, Victoria, Australia

${ }^{26}$ These authors contributed equally to this work.

*Correspondence: Professor T Eggermann, Institute of Human Genetics, University Hospital, RWTH Aachen, Pauwelsstr. 30 , Aachen 52074 , Germany. Tel: +49 241 8088008; Fax: +49 214 8082394; E-mail: teggermann@ukaachen.de

Received 15 July 2015; revised 3 September 2015; accepted 11 September 2015; published online 28 October 2015
} 


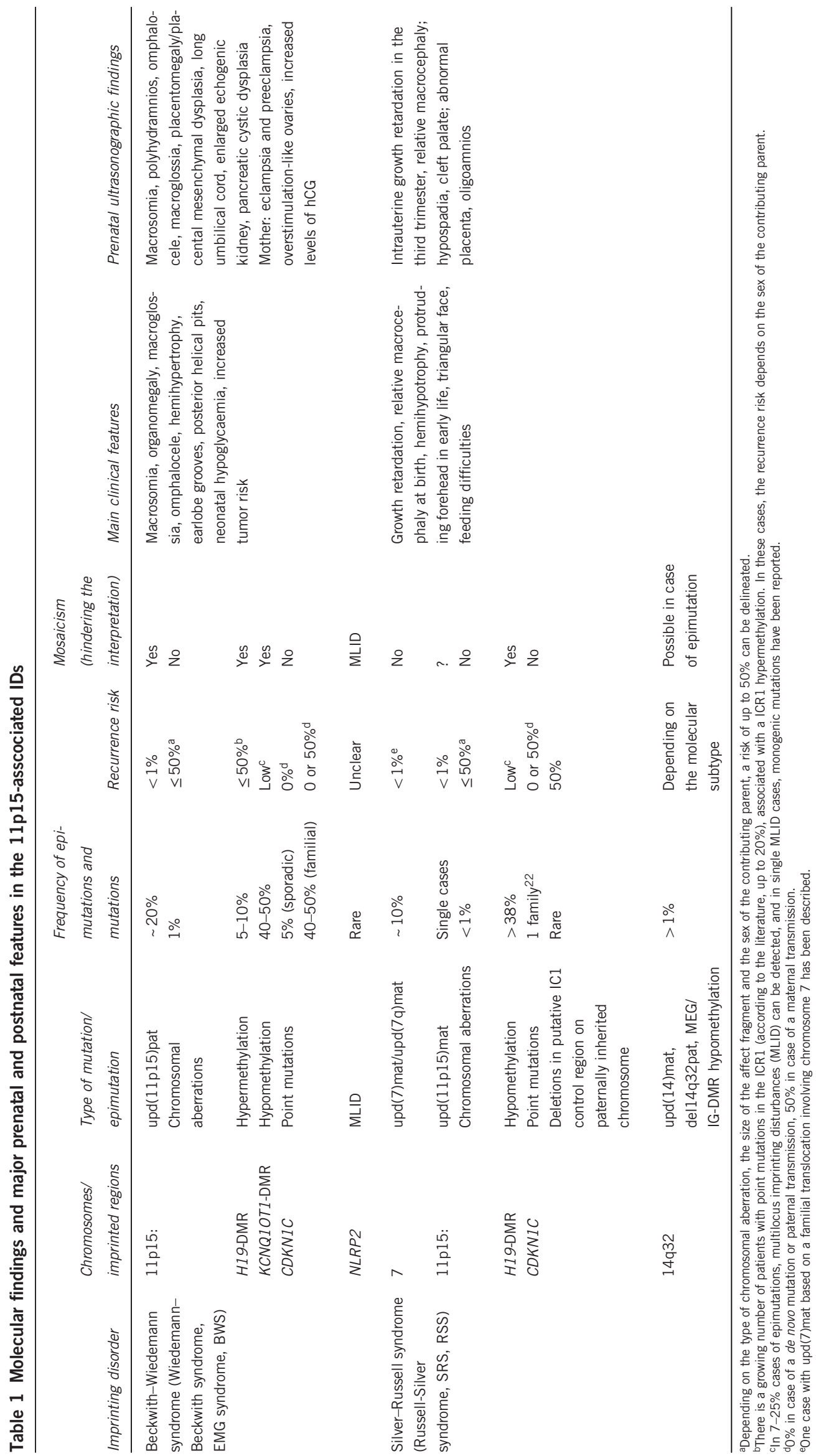


imprinting control regions (ICRs): the imprinting control region 1 (ICR1) is methylated on the paternal allele and regulates the maternally expressed H19 and paternally expressed IGF2 genes via the $H 19$ differentially methylated region (H19-DMR), whereas the maternally methylated KCNQ1OT1-DMR in the ICR2 regulates the maternally expressed CDKN1C and paternally expressed KCNQ1OT1 genes.

In BWS, both epimutations and genomic alterations affecting 11 p15.5 are present in $>70 \%$ of patients, whereas up to $60 \%$ of SRS patients carry 11p15.5 disturbances (Table 1). Additionally, 10\% of the latter patients show a maternal uniparental disomy of chromosome 7 (upd(7)mat). Furthermore, in patients exhibiting features of the two disorders aberrations not involving chromosomes 7 and $11 \mathrm{p} 15.5^{6-9}$ are reported.

Because of the methodological improvement and the growing knowledge on 11p15-associated IDs, there is an increasing demand not only for postnatal confirmation of a clinical diagnosis but also for prenatal testing. This request might also be boosted by an altered reproductive behavior at least in developed countries: both advanced maternal age at childbirth as well as assisted reproduction technologies (ART) have been suggested as predisposing factors for IDs. Advanced maternal age might promote the development of chromosomal aberrations, including uniparental disomy, ${ }^{10,11}$ whereas the ART procedure itself might influence the establishment and/or maintenance of imprinting marks. ${ }^{12}$

However, the complex molecular findings and alterations in BWS and SRS patients are a challenge for laboratories and clinical geneticists offering these tests. They have to consider a wide range of detectable disturbances, occurrence of mosaicism, limitations of the applicable tests, consequences for the patients, the families and - in the case of prenatal testing - the informative value of the test results. Based on their experiences and work in the field of 11p15.5-associated disorders, the authors of this paper are aware of these challenges and summarize the current knowledge on prenatal testing in BWS and SRS.

\section{MOLECULAR ALTERATIONS IN IDS AND MOSAICISM}

Similar to other IDs, distinct molecular sub-groups can be detected in BWS and SRS: (a) (micro)chromosomal deletions, duplications and rearrangements; (b) mutations in imprinted genes or their imprinting control regions (ICR); (c) uniparental disomy (UPD); and (d) epimutations (ie, aberrant methylation without alteration of the genomic DNA sequence).

Chromosomal or intragenic mutations affecting imprinted loci (a, b) can occur de novo, but in many cases familial inheritance has been reported. UPD (c) and epimutations (d) do not affect the genomic DNA sequence itself, but the regulation of gene expression by parent-of-origin imprinting. All subgroups affect the fine-tuned expression of genes controlled by ICRs.

When UPDs and epimutations arise postzygotically during early embryonic cell divisions, they are most likely associated with a mosaic distribution (Table 1). However, mosaicism has also been reported for genomic alterations. ${ }^{13}$ Mosaicism can disturb genotype-phenotype correlations and may explain, in part, the broad phenotypic range associated with these disorders (Table 1). From the diagnostic point of view, it can significantly hamper the detection of the molecular alteration (eg, Alders et $\mathrm{al}^{14}$ ). Therefore, it is in principle impossible to exclude the clinical diagnosis in case of a negative test result, because other untested tissues might carry the alteration.

\section{GENOMIC MUTATIONS AND EPIMUTATIONS IN BWS AND SRS}

Both SRS and BWS are mainly associated with molecular alterations affecting the two ICRs in 11p15.5 (Table 1). The molecular defects in $11 \mathrm{p} 15.5$ in the two disorders comprise the four different types of mutations and epimutations described before (for a review, see Shuman et $a l^{15}$ and $\mathrm{Saal}^{16}$ ). In patients with SRS features, a considerable number of cases carry a upd(7)mat or (submicroscopic) changes of different chromosomes. ${ }^{17,18}$ Furthermore, the number of SRS patients with reported molecular defects in $14 \mathrm{q} 32$ corresponding to Temple syndrome (TS14) is increasing. ${ }^{6,7,19}$ For patients with BWS features, aberrations of other chromosomal regions and genes have rarely been reported. ${ }^{8,9,20}$

Up to $60 \%$ of SRS patients show a DNA hypomethylation of the ICR1 in 11p15; in addition, in single cases complete or partial maternal duplications of $11 \mathrm{p} 15$ have been identified (for a review, Begemann et $\left.a^{21}\right)$. Other disturbances of chromosome $11(\operatorname{upd}(11)$ mat; CDKN1C, IGF2 point mutations) are rare in SRS, and the gene variants only occur in familial cases. ${ }^{22-25}$

In BWS, the opposite epigenetic or genetic changes in $11 \mathrm{p} 15$ have been observed in nearly $70 \%$ of patients with a preponderance of DNA hypomethylation at the ICR2 accounting for up to $50 \%$ of all BWS cases. Upd(11p15)pat is the second most common alteration ( 20\%), while DNA hypermethylation at ICR1 is rare (5-10\%). In up to $5 \%$ of sporadic and $50 \%$ of familial BWS patients, CDKN1C point mutations can be identified. Additionally, point mutations and small deletions at protein-binding sites within the ICR $1^{26}$ as well as microdeletions within the ICR $1^{27}$ or of the complete ICR $2^{28,29}$ are observed. In these families, BWS follows autosomal-dominant inheritance with incomplete penetrance dependent on the sex of the parent contributing the mutation. For both IDs, a growing number of cases and families with small rearrangements and imbalances restricted to single DMR in either the ICR1 or ICR2 have been reported. ${ }^{21}$

Despite the initial correlation between 11p15 in BWS and 11p15 and chromosome 7 in SRS, a growing number of phenotypically related patients are described displaying hypomethylation of imprinted loci additional to the disease-specific ones. This multilocus imprinting disturbance (MLID) is detectable in 7\% of SRS patients with ICR1 and $25 \%$ of BWS patients with ICR2 hypomethylation. Monogenic mutations have been reported that predispose to MLID, ${ }^{30-32}$ thus the prediction of a recurrence risk is difficult in patients carrying MLID.

\section{CLINICAL FINDINGS IN BWS AND SRS}

The most prominent clinical feature in both IDs is growth disturbance. Indeed, the overgrowth in BWS and the growth restriction in SRS impressively reflect the underlying opposite molecular defects (Table 1). In both syndromes, the clinical diagnosis is often difficult owing to their variable presentation during childhood and adolescence, and the molecular and phenotypic findings overlap with other IDs and syndromes. ${ }^{15,16}$ Thus differential diagnoses should always be considered, in particular in the course of a (prenatal) diagnostic workup.

\section{Beckwith-Wiedemann syndrome}

BWS was originally referred to as EMG syndrome because of its three cardinal features: Exomphalos, Macroglossia and (neonatal) macrosomia (Gigantism). A recognizable facial gestalt of BWS is common and may include prominent eyes with intraorbital creases, facial naevus flammeus, midfacial hypoplasia, full lower lips, earlobe creases and posterior helical pits. Additional clinical features of BWS include neonatal hypoglycemia, hemihyperplasia, organomegaly (heart, liver, 


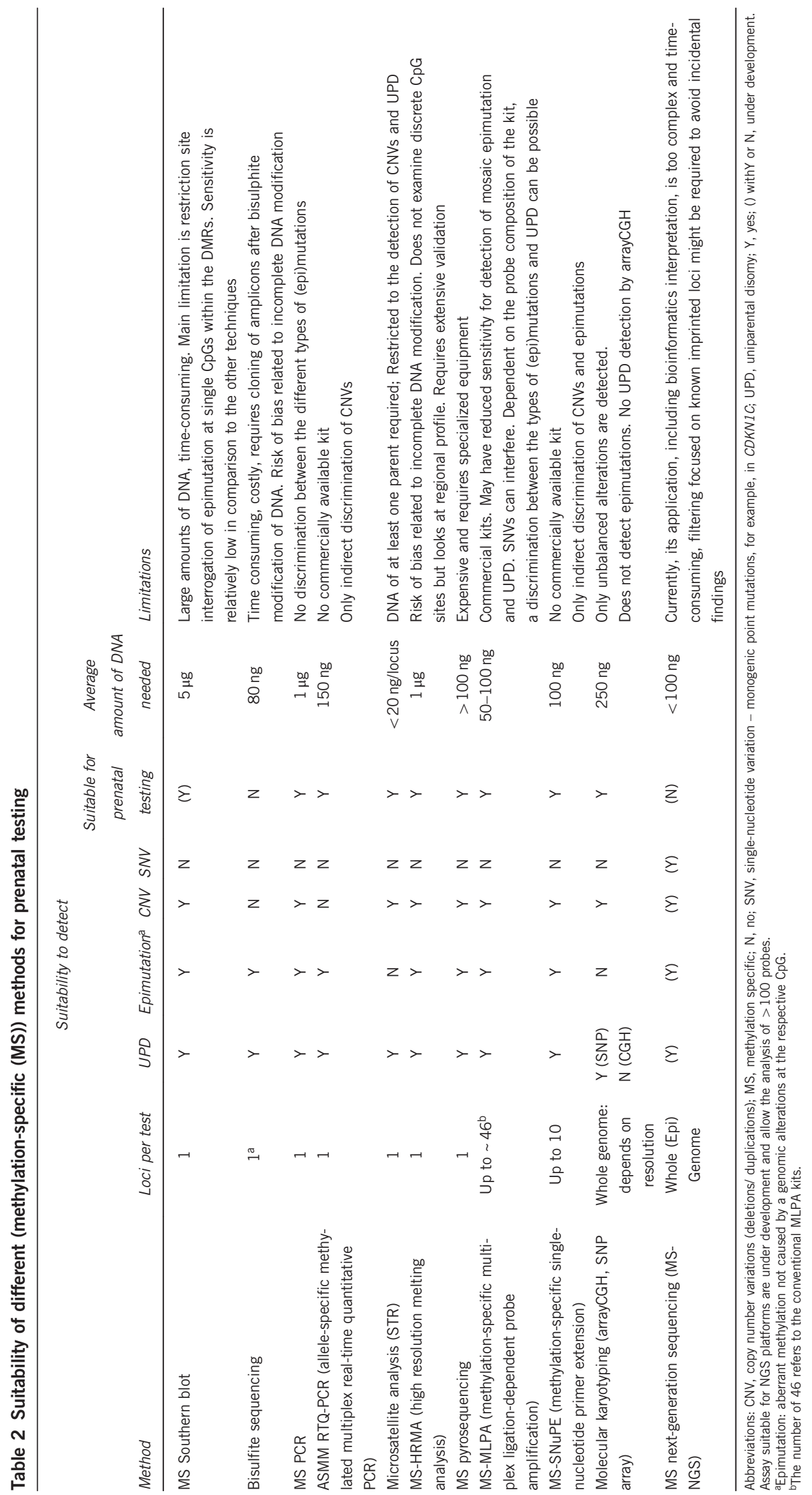


spleen, pancreas, kidneys, adrenals) and unilateral or bilateral renal abnormalities (eg, medullary dysplasia, nephrocalcinosis, medullary sponge kidney) (Table 1). In 5-7\% of children with BWS, embryonal tumors (most commonly: Wilms tumor, hepatoblastoma, neuroblastoma, and rhabdomyosarcoma) are diagnosed. Early death may occur from complications of prematurity, hypoglycemia, cardiomyopathy, macroglossia or tumors. Growth rate slows around age 7-8 years. The tumor risk also reduces beyond this age, but in case of upd(11)pat a mosaic genome-wide paternal uniparental diploidy ${ }^{33}$ has to be excluded as these patients have a lifelong risk to develop tumors. Hemihyperplasia may affect segmental regions of the body or selected organs and tissues. Prenatally, the major signs detectable by ultrasound are placental mesenchymal dysplasia, ${ }^{34}$ omphalocele (detectable after the thirteenth week of pregnancy (wp)), polyhydramnios and fetal macrosomia (detectable after the nineteenth wp) (Table 1). ${ }^{35}$ The incidence of polyhydramnios, premature birth and fetal macrosomia in BWS has been estimated as $\sim 50 \%$ (for gene reviews, see Shuman et $a l^{15}$ ). Other common features include a long umbilical cord and an enlarged placenta that averages almost twice the normal weight for gestational age. During pregnancy, preeclampsia or eclampsia may occur. ${ }^{35,36}$

\section{Silver-Russell syndrome}

SRS is characterized by intrauterine and postnatal growth restriction with a typical facial gestalt (for a review, Saal ${ }^{16}$ ). In the majority of patients, birth weight is 2-3 s.d. below the mean, and growth restriction persists. As a result, mean adult height without $\mathrm{GH}$ treatment is $150 \mathrm{~cm}$ in males and $139 \mathrm{~cm}$ in females. ${ }^{37}$ Head circumference can be normal, thus the patients show a relative macrocephaly. Clinodactyly V and face/body asymmetry (hemihypoplasia) are frequent. The typical facies include a prominent forehead, a triangular face and a pointed chin. Children with SRS might exhibit a (slight) developmental delay (both motor and cognitive) and learning disabilities, however, these features predominantly occur in carriers with upd(7)mat. ${ }^{38}$ Complications of SRS include gastrointestinal reflux and failure to thrive. Children may benefit from GH treatment. Intrauterine growth retardation and relative macrocephaly usually occur in the third trimester and are often the only clinical features of SRS to be detected by fetal ultrasonography. In rare cases, hypospadia and/or cleft palate are reported associated with a very small placenta.

\section{RECURRENCE RISK ESTIMATION IN BWS AND SRS}

The majority of cases with BWS and SRS have been reported to occur sporadically; this is reflected by the type of (epi)mutations in both disorders: ICR1 hypomethylation in SRS as well as ICR2 hypomethylation and upd(11)pat in BWS mainly occur as mosaicism and probably originate from postzygotic errors. In contrast, constitutional mutations (point mutations, duplications/deletions) are associated with a significantly increased recurrence risk of up to $50 \%$ depending from the affected paternal allele. However, with our increased knowledge about molecular disturbances in both syndromes, we must adjust risk estimation and counseling. Furthermore, specific mutations have been identified that cause aberrant methylation either by acting in cis (eg, small deletions in ICR $\left.1^{39}\right)$ or in trans $\left(N L R P 2^{32}\right)$ In particular, approximately $20 \%$ of BWS patients with ICR1 hypermethylation have point mutations or small deletions in OCT4/SOX2-binding sites within the H19-DMR. ${ }^{26}$

Thus, to offer genetic counseling to families with IDs, the knowledge of the nature of the mutation or epimutation subtype is essential to delineate exact risk figures, and genetic counseling by an experienced clinical geneticist is emphasized.

\section{MOLECULAR TESTING FOR BWS AND SRS}

The currently applied molecular tests in ID diagnostics are mainly restricted to single disease-specific loci, but technical, biological and clinical factors influence the diagnostic yield. An increasing number of molecular techniques are available (Table 2) either targeting only one imprinted region or several DMRs. The current lack of standardization can make comparisons between molecular results from different laboratories problematic, furthermore some of the applied methods do not allow the discrimination between the different types of (epi) mutations (Table 2). Several laboratories performing clinical testing for BWS and SRS already participate in informal sample exchange programs or have extensively validated several different testing approaches. Additionally, the European Molecular Quality Network (EMQN) has established a BWS/SRS quality assessment scheme for 11p15.5-associated IDs.

A major challenge is mosaicism (eg, Azzi et a ${ }^{40}$ ). In case of a lowlevel mosaicism, a false-negative result will be generated. Thus, in case of an unequivocal diagnosis and negative testing results in DNA derived from blood, another tissue (fibroblasts, buccal cells) should be tested.

Another problem of diagnostic testing for IDs is the broad spectrum of molecular alterations: an imprinted locus can be affected by different types of (epi)mutations. Additionally in some IDs even different loci might be affected. Therefore, the diagnostic workup for each ID requires a profound knowledge on the possible molecular findings and the limitations of the applied (Tables 1 and 2). For putative variants, additional laboratory tests (eg, molecular karyotyping, FISH, genotyping) might be required; for point mutations, a bioinformatics estimation of its pathogenicity has to be performed, along with family studies to determine the mode of transmission and segregation with phenotype.

It is foreseeable that the increasing implementation of nextgeneration sequencing (NGS)-based assays in routine diagnostics will also find the way in the diagnostic workup of IDs. NGS has the potential to detect all types of (epi)mutations at once and can target multiple loci; massive parallel sequencing will also allow a more sensitive mosaic detection. However, future non-invasive prenatal testing (NIPT) of IDs will probably be restricted to families with chromosomal rearrangements. Prenatal DNA methylome profiling or transcriptomic analysis might indeed result in the identification of biomarkers in the early diagnosis of placental dysfunction or aneuploidies and other developmental defects, ${ }^{41,42}$ but it might not be suitable for NIPT of IDs owing to the early time of sample drawing and the multiple challenges in determining the fetal methylome/ transcriptome status in maternal plasma.

\section{EXPERIENCES FROM PRENATAL BWS AND SRS TESTING}

In the centers contributing to this review, 160 prenatal tests have been performed (Tables $3 \mathrm{a}-\mathrm{c}$ ), 122 for BWS and 38 for SRS. In this cohort, several criteria have been evaluated, including the methods applied, the tissues analyzed (chorionic villus (CVS), amniocytic fluid cells (AF) or fetal blood; cultured and native cells), the reasons for referral, the number of positively tested cases and the number of false-positive/ negative results (Tables $3 \mathrm{a}-\mathrm{c}$ ).

\section{Methods}

Different methods are applied (Table 3a), with different sensitivities and limitations with respect to the discrimination between the different molecular subtypes (Table 2). However, as shown for the imprinted GNAS locus, the heterogeneity of molecular tests does not affect the correctness of the molecular diagnosis. ${ }^{43}$ Thus no specific 

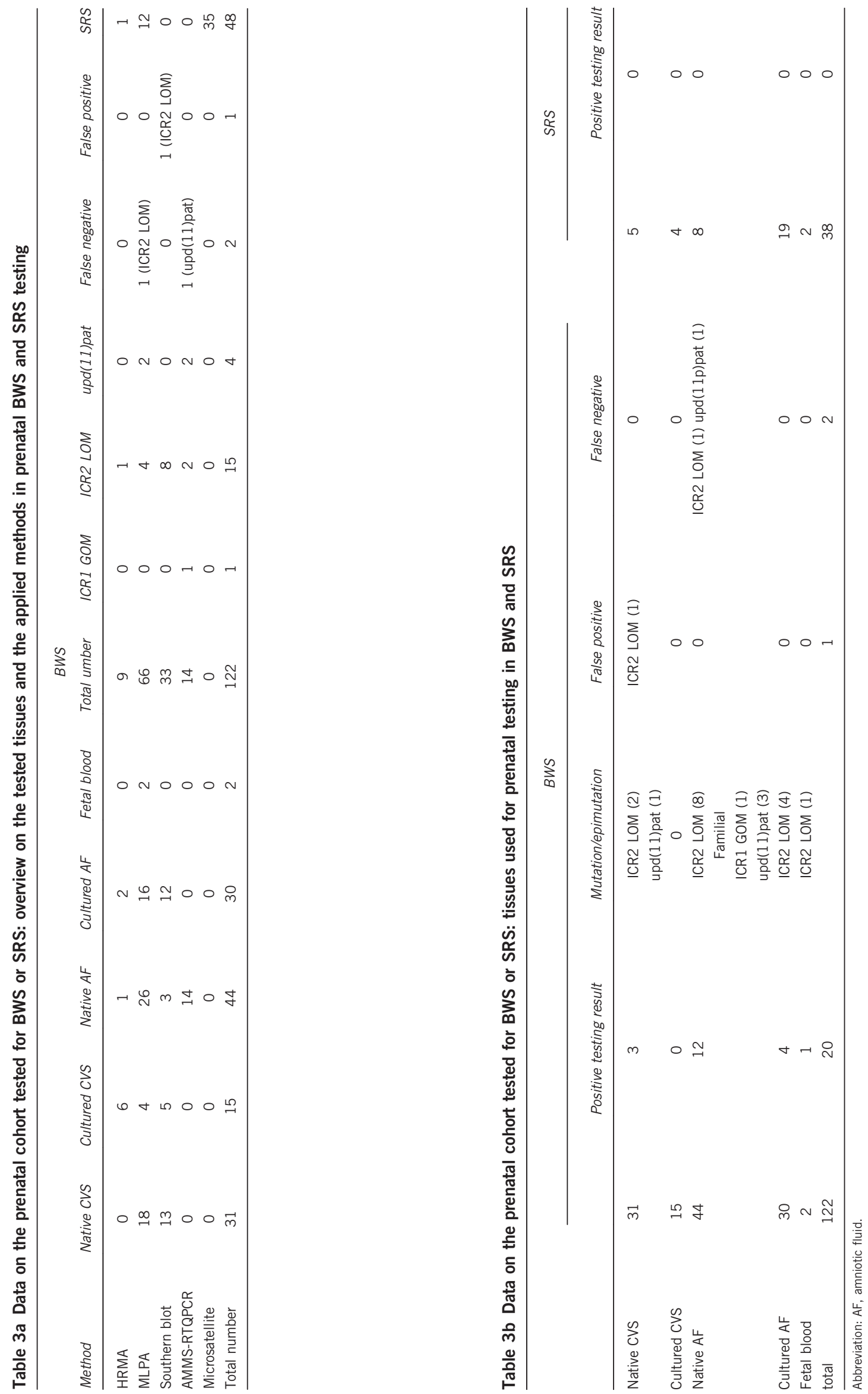
assay can be recommended, but in any case a careful validation has to be carried out and advantages and disadvantages in the context of prenatal testing (duration of the protocol, amount of DNA) have to be considered. For the methods analyzed by our centers, between 50 (MS-MLPA) and 100 (HRMA, AMMS-RTqPCR) control samples have been used for validation. Furthermore, the limitations of the different tests have to be taken into account, for example, microsatellite typing can be peformed to determine UPD, but this method does, of course, not exclude epimutations.

\section{Prenatal tissue}

Both native and cultured prenatal cells might be suitable for molecular testing (Tables 3a and b). However, it should be kept in mind that cell culture might result in clonal features that do not always correlate with the true biological status of the fetus/placenta. Furthermore, in the case of early CVS (before $12 \mathrm{wp}$ ) the imprinting marks at the loci of interest might not be finally set. ${ }^{44}$ As CVS is an extraembryonic tissue, it might not reflect the (epi)genetic constitution of the fetus. Although the analysis of cells derived from the fetus itself offers the best chance to detect altered imprinting marks, our data show that even in this case the risk of false-positive results cannot be prevented (Table $3 \mathrm{~b}$ ). In general, mosaicism is a major challenge in molecular testing of BWS and SRS, and it can never be excluded.

\section{Reasons for referral for prenatal testing}

As the experiences from our centers show, the reasons for referring prenatal samples for testing differ remarkably between BWS and SRS (Table 3c). A positive family history is generally a rare indication for both disorders and is generally restricted to monogenic mutations (eg, CDKN1C) or chromosomal rearrangements (eg, 11p duplications). In BWS, abnormal ultrasound (ie, abdominal wall defects/ omphalocele) is the main reason for invasive prenatal testing. Although IUGR is the key prenatal clinical feature of SRS, it is a rare indication for prenatal testing, presumably because it becomes obvious only in the third trimester and is generally an unspecific symptom, which should be regarded with caution as an indication for prenatal testing. In prenatal SRS testing, chromosomal aberrations (trisomy 7 in CVS, familial translocations) are the predominant indications.

\section{Prenatal testing results and pregnancy outcome}

The results of prenatal testing for BWS and SRS reflect both the clinical heterogeneity and the molecular complexity of the disorders (Tables $3 \mathrm{a}$ and $\mathrm{b}$ ). In the case of SRS, IUGR is the most obvious ultrasound sign but very unspecific and can therefore merely be regarded as a reliable feature for prenatal testing. This might explain why no positive cases have yet been ascertained prenatally. Indeed, the major reasons for prenatal testing for SRS are chromosomal aberrations, that is, familial translocations involving chromosomes 7 and 11 or trisomy 7 in CVS.

The distribution of positive test results in the cases ascertained for BWS testing reflects the published frequencies of (epi)mutations (Tables $3 \mathrm{a}$ and $\mathrm{b}$ ). The ICR2 hypomethylation accounts for the majority $(n=15)$ of the 20 positive test results, 4 fetuses carried upd (11)pat and 1 ICR1 hypermethylation. In 32 fetuses, prenatal CDKN1C sequencing has been performed. In two fetuses, the mothers had been identified as mutation carriers. In the other cases, ultrasound findings were indicative for BWS. In that group, four fetuses carried pathogenic mutations, in one the mutation was maternal in origin, too. 
False-positive/negative cases

In one case initially diagnosed as ICR2 hypomethylation in CVS, the analysis of a second prenatal tissue (amniotic fluid, AF) and the clinical outcome revealed a false-positive testing result. These discrepant findings can be explained by an incomplete methylation at the time of sample drawing $(11+6 \mathrm{wp})$. In two cases with a negative prenatal test result, postnatal testing identified ICR2 hypomethylation and upd(11)pat, respectively, thereby confirming the prenatal suspicion of BWS. This discrepancy is in accordance with a previous report on the mosaic distributions of molecular disturbances in BWS. ${ }^{14}$ The example vividly illustrates that laboratories offering prenatal (and postnatal) tests have to be aware of and to report on the risk to miss 11 p15 defects owing to somatic mosaicism and the value of prenatal testing has to be critically discussed with the families.

\section{CHALLENGES FOR PRENATAL TESTING IN BWS AND SRS}

In general, prior to molecular prenatal testing, genetic counseling should be offered to discuss the informativity and limitations of prenatal ID testing (Figure 1) and finally the consequences of both positive and negative test results.

First of all, general issues of prenatal (molecular) have to be considered, such as the individual situation (risk and suitability of the invasive test for the current pregnancy), the risk of contamination of the fetal sample with maternal cells resulting in a false-negative result

\section{Indications}

- Family history, an inherited copy number variation/chromosomal translocation or genomic mutation involving the $11 \mathrm{p}$ region.

- Chromosomal aberration (trisomy in CVS; (familial) chromosomal rearrangement involving a chromosome carrying imprinted genes related to BWS/SRS (chromosomes 11 (SRS and BWS) and 7 (SRS)).

- Abnormal ultrasound findings: BWS: omphalocele, macroglossia, visceromegaly, enlarged adrenals, macrosomia with no obvious mechanism (e.g. maternal diabetes mellitus). SRS: IUGR is sometimes regarded as an indication but the symptom alone should not be an indication.

Tissue

- CVS and cultured amniocytes can be used for copy number (CNV)/mutations analysis and upd(7)mat. - In case of upd(11) and epimutations, native amniotic fluid is the preferred tissue. Amniocytes may show clonal features that not always correlated with the true biological status of the fetus/placenta, in case of early CVS the imprinting marks at the loci of interest might not be finally set.

- Maternal contamination: a parallel determination of maternal and fetal STRs (microsatellites) should be warranted in any prenatal study.

- Which quantity of amniotic fluid/DNA is needed for the test?

Methods:

- Is the method suitable for prenatal testing?

- Is it rapid? (in case of molecular prenatal testing, results are expected within 3-5 days)

- Does it detect the molecular alteration of interest? The lab should be aware that a substantial number of fetuses will have mosaicism. It has to be checked if the applied method warrants that at least high and moderate degrees of mosaicisms are observed.

- Has it been validated (in-house)?

- What are the limitations?

- What is the sensitivity for mosaic detection?

\section{Molecular Findings and their interpretation}

- Type of mutation: UPD, CNV, epimutation, point mutation

- Mosaicism: In case of a positive testing result: the suspected diagnosis can be confirmed, but a prediction of the phenotypic outcome is not possible but might be delineated from the ultrasound findings. In case of a negative testing result: the mosaic presence of UPD or epimutations can never be excluded. Mosaicism in case of constitutional mutations (CNVs, monogenic point mutations) can be neglected.

- How to interpreter the results in case of a twin pregnancy? (the majority of monozygotic twins are clinically discordant)

\section{Consequence/Ethical issue}

- The clinical outcome is difficult to predict, and is rather based on the ultrasound findings than on the molecular testing results.

- What are the consequences for the continuation of the pregnancy in case of a positive or negative testing result?

- Does the positive testing help to continue the pregnancy because it explains the prenatal abnormalities but both syndromes are associated with relatively mild features? See below - Does the positive test result in an induced abortion? Are BWS or SRS direct or indirect reasons for therapeutic abortions?

Figure 1 Considerations to be addressed prior to prenatal testing of BWS and SRS. 
(a general hazard of invasive prenatal testing), the suitability of the invasively drawn sample for the applied test (amount and quality of the DNA) and the difficult phenotype prediction in the case of a positive testing result.

Furthermore, ID-specific considerations have to be addressed:

- The timing of sample drawing and the methylation status of the DMR of interest at that time. It is well known that some DMRs are not finally established during the time of CVS in the 10-12th wp, ${ }^{44}$ and methylation-specific testing at that time leads to false results, explaining the false-positive case in our cohort (Tables $3 \mathrm{a}$ and $\mathrm{b}$ ).

- Mosaicism can cause a false-negative testing result as shown in our study cohort (Tables 3a-c).

- So far, there is no consensus on the target methylated CpGs and DMRs in ID testing, therefore the experiences and data are scarcely comparable between different laboratories, and every test has to be validated separately in-house.

- Because of the heterogeneity and complexity of the molecular findings in the 11p15-associated IDs, unusual molecular alterations may elude identification. Additionally, the informative content of the available assay are different and might hamper the diagnostic process (Table 2).

Whereas these aspects address the laboratory workup and are the basis for a reliable test result, the handling of both negative and positive results are probably in the purview of the genetic counseling session. However, the technical limitations should also be included in the written report.

\section{A positive prenatal test result}

In the rare case of a positive family history, the families are generally aware of the significance of a positive testing result. The situation is different for the majority of couples who are confronted with the possibility of giving birth to a child with BWS or SRS for the first time. In this situation, the molecular workup might help to confirm a clinical diagnosis and exclude other entities, in particular those associated with intellectual disabilities. In all such pregnancies, detailed ultrasound at least at 18-20 wp and again at 25-28 wp should be directed to assess growth parameters and the presence of abdominal wall defects, organomegaly, renal and cardiac abnormalities, cleft palate and macroglossia. Early prenatal confirmation of a clinical diagnosis can result in recommendation for hospital delivery, monitoring the baby for hypoglycemia, surveillance for tumors, further therapeutic options and support of the parents. Both BWS and SRS babies may have feeding difficulties in the neonatal and early childhood period.

Nevertheless, in some circumstances a positive test result may lead to a decision to terminate the pregnancy. Vice versa, a positive diagnostic test might result in a therapeutic abortion being avoided for example, in a fetus with an anterior wall defect the molecular diagnosis of BWS implies that the risk of learning disability is small. Indeed, some of the contributing authors are aware of pregnancies with a negative molecular testing, which were then terminated because of the uncertainty of the underlying cause of the clinical finding.

\section{A negative prenatal test result}

As already discussed, the major molecular alterations both in BWS and SRS (ie, epimutations in SRS/BWS and upd(11)pat in BWS) can occur in the mosaic form ${ }^{14,21}$ and might escape molecular detection because of the low level of cells harboring the disturbance. The authors are aware of two false-negative prenatal testing result with an epimutation detected after birth (Table 3b). Hence, a normal prenatal test result cannot absolutely exclude a diagnosis of BWS and SRS. Furthermore, as previously indicated, unknown genes and mechanisms are likely to exist in addition to the mosaic cases that escape detection using current testing approaches. These issues need to be emphasized in genetic counseling and in clinical reports.

\section{Postnatal testing}

In case of a persisting clinical suspicion of SRS and BWS after a negative prenatal testing result, postnatal testing should also be performed to confirm any findings. Indeed, the limitations of prenatal testing need to be very clear prior to testing being undertaken.

\section{CONCLUSION}

The growing knowledge on the molecular basis of BWS and SRS and the increasing number of positively tested patients results in an increasing demand for prenatal testing for these diseases. Before offering these tests, numerous questions have to be tabled and discussed with the couple, ranging from methodological questions to the ethical topics as listed in Figure 1. In particular, in case of a negative testing result, its informative value has to be critically discussed. However, the optimization of tests and their implementation as well as the current quality assessment schemes under development by the EMQN for BWS and SRS will contribute to a more reliable and meaningful prenatal diagnosis. Thus the decision on the continuation of a pregnancy suspicious for BWS or SRS has to take into account all these considerations, but finally it has to be reached by the parents after a self-determined decision process and according to the national laws.

\section{CONFLICT OF INTEREST}

The authors declare no conflict of interest.

\section{ACKNOWLEDGEMENTS}

The authors are members of the COST Action BM1208 and EUCID.net (European congenital imprinting disorders network; http://www.imprintingdisorders.eu). TE, MB, LS, KE and DP are supported by the Bundesministerium für Bildung und Forschung (Network 'Imprinting Diseases', 01GM1513B/C) and KC and MKW by National Science Centre project nos. 1149/B/P01/2011/40 (NN407114940) and 2853/B/P01/2010/39 (NN407285339). PL is supported by a Grant of the Ministry of Science (ISCIII, PI 11/02491). FB and IN are funded by INSERM, UPMC Paris 6 funding and EPIFEGRO2010 (Novonordisk grant). SR and LL are funded by the Ministero della Salute (IT) (08C207_2012).

\footnotetext{
1 Reik W, Walter J: Genomic imprinting: parental influence on the genome. Nat Rev Genet 2001; 2: 21-32.

2 Mackay DJ, Eggermann T, Buiting $\mathrm{K}$ et al: Multilocus methylation defects in imprinting disorders. Biomol Concepts 2015; 6: 47-57.

3 Eggermann T, Netchine I, Temple IK et al: Congenital imprinting disorders: EUCID.net a network to decipher their aetiology and to improve the diagnostic and clinical care. Clin Epigenetics 2015; 7: 23.

4 Gabory A, Ripoche M-A, Le Digarcher A et al: H19 acts as a trans regulator of the imprinted gene network controlling growth in mice. Development 2009; 136: 3413-3421.

5 Sandhu KS, Shi C, Sjölinder M et al: Nonallelic transvection of multiple imprinted loci is organized by the $\mathrm{H} 19$ imprinting control region during germline development service. Genes Dev 2009; 23: 2598-2603.

6 Eggermann T, Heilsberg AK, Bens $S$ et al: Additional molecular findings in 11p15associated imprinting disorders: an urgent need for multi-locus testing. J Mol Med (Berl) 2014; 92: 769-777.

7 Azzi S, Salem J, Thibaud N et al: A prospective study validating a clinical scoring system and demonstrating phenotypical-genotypical correlations in Silver-Russell syndrome. J Med Genet 2015; 52: 446-453.
} 
8 Naik S, Riordan-Eva E, Thomas NS et al: Large de novo deletion of 7p15.1 to 7p12.1 involving the imprinted gene GRB10 associated with a complex phenotype including features of Beckwith Wiedemann syndrome. Eur J Med Genet 2011; 54: 89-93.

9 Brewer CM, Lam WW, Hayward C, Grace E, Maher ER, FitzPatrick DR: BeckwithWiedemann syndrome in a child with chromosome 18q deletion. J Med Genet 1998; 35: 162-164.

10 Cho SY, Ki CS, Sohn YB et al: The proportion of uniparental disomy is increased in Prader-Willi syndrome due to an advanced maternal childbearing age in Korea. J Hum Genet 2013; 58: 150-154.

11 Matsubara K, Murakami N, Nagai T, Ogata T: Maternal age effect on the development of Prader-Willi syndrome resulting from upd(15)mat through meiosis 1 errors. J Hum Genet 2011; 56: 566-571

12 Vermeiden JP, Bernardus RE: Are imprinting disorders more prevalent after human in vitro fertilization or intracytoplasmic sperm injection? Fertil Steril 2013; 99: 642-651.

13 Beygo J, Elbracht M, de Groot $\mathrm{K}$ et al: Novel deletions affecting the MEG3-DMR provide further evidence for a hierarchical regulation of imprinting in 14q32. Eur J Hum Genet 2015; 23: 180-188

14 Alders M, Maas SM, Kadouch DJ et al: Methylation analysis in tongue tissue of BWS patients identifies the (EPI) genetic cause in 3 patients with normal methylation levels in blood. Eur J Med Genet 2014; 57: 293-297.

15 Shuman C, Beckwith JB, Smith AC, Weksberg R. Beckwith-Wiedemann Syndrome. In Pagon RA, Adam MP, Ardinger HH et al (eds): GeneReviews(R). Seattle, WA, USA: University of Washington, Seattle University of Washington, All rights reserved 1993.

16 Saal HM: Russell-Silver Syndrome. In: Pagon RA, Adam MP, Ardinger HH et al (eds): GeneReviews(R). Seattle, WA, USA: University of Washington, Seattle University of Washington, All rights reserved. 1993.

17 Bruce S, Hannula-Jouppi K, Puoskari M et al: Submicroscopic genomic alterations in Silver-Russell syndrome and Silver-Russell-like patients. J Med Genet 2010; 47: 816-822.

18 Spengler S, Begemann M, Ortiz Bruchle $\mathrm{N}$ et al: Molecular karyotyping as a relevant diagnostic tool in children with growth retardation with Silver-Russell features. J Pediatr 2012; 161: 933-942.

19 Kagami M, Mizuno S, Matsubara K et al: Epimutations of the IG-DMR and the MEG3DMR at the $14 \mathrm{q} 32.2$ imprinted region in two patients with Silver-Russell syndromecompatible phenotype. Eur J Hum Genet 2014; 23: 1062-1067.

20 Fryns JP, Kleczkowska A, Devriendt K, Devliegher H, Van den Berghe H: WiedemannBeckwith syndrome and chromosomal duplication 4q/deficiency 18p. Genet Couns 1993; 4: 37-41.

21 Begemann M, Spengler S, Gogiel M et al: Clinical significance of copy number variations in the $11 \mathrm{p} 15.5$ imprinting control regions: new cases and review of the literature. J Med Genet 2012; 49: 547-553.

22 Brioude F, Oliver-Petit I, Blaise A et al: CDKN1C mutation affecting the PCNA-binding domain as a cause of familial Russell Silver syndrome. J Med Genet 2013; 50: 823-830.

23 Joyce CA, Sharp A, Walker JM, Bullman H, Temple IK: Duplication of 7p12.1-p13, including GRB10 and IGFBP1, in a mother and daughter with features of Silver-Russell syndrome. Hum Genet 1999; 105: 273-280.

24 Bullman H, Lever M, Robinson DO, Mackay DJ, Holder SE, Wakeling EL: Mosaic maternal uniparental disomy of chromosome 11 in a patient with Silver-Russell syndrome. J Med Genet 2008; 45: 396-399.

25 Begemann M, Zirn B, Santen G et al: Paternally inherited IGF2 mutation and growth restriction. N Engl J Med 2015; 373: 349-356.

26 Abi Habib W, Azzi S, Brioude F et al: Extensive investigation of the IGF2/H19 imprinting control region reveals novel OCT4/SOX2 binding site defects associated with specific methylation patterns in Beckwith-Wiedemann syndrome. Hum Mol Genet 2014; 23: 5763-5773.

27 Beygo J, Citro V, Sparago A et al: The molecular function and clinical phenotype of partial deletions of the IGF2/H19 imprinting control region depends on the spatial arrangement of the remaining CTCF-binding sites. Hum Mol Genet 2013; 22: 544-557.

28 Niemitz EL, DeBaun MR, Fallon J et al: Microdeletion of LIT1 in familial Beckwith Wiedemann syndrome. Am J Hum Genet 2004; 75: 844-849.

29 Algar E, Dagar V, Sebaj M, Pachter N: An 11p15 imprinting centre region 2 deletion in a family with Beckwith Wiedemann syndrome provides insights into imprinting control at CDKN1C. PLoS One 2011; 6: e29034-e29034.

30 Murdoch S, Djuric U, Mazhar B et al: Mutations in NALP7 cause recurrent hydatidiform moles and reproductive wastage in humans. Nat Genet 2006; 38: 300-302.

31 Mackay DJG, Callaway JLa, Marks SM et al: Hypomethylation of multiple imprinted loci in individuals with transient neonatal diabetes is associated with mutations in ZFP57. Nat Genet 2008; 40: 949-951.

32 Meyer E, Lim D, Pasha S et al: Germline mutation in NLRP2 (NALP2) in a familial imprinting disorder (Beckwith-Wiedemann Syndrome). PLoS Genet 2009; 5: e1000423-e1000423.

33 Kalish JM, Conlin LK, Bhatti TR et al: Clinical features of three girls with mosaic genome-wide paternal uniparental isodisomy. Am J Med Genet A 2013; 161a: 1929-1939.

34 Pham T, Steele J, Stayboldt C, Chan L, Benirschke K: Placental mesenchymal dysplasia is associated with high rates of intrauterine growth restriction and fetal demise: a report of 11 new cases and a review of the literature. Am J Clin Pathol 2006; 126: $67-78$.

35 Kagan KO, Berg C, Dufke A, Geipel A, Hoopmann M, Abele H: Novel fetal and maternal sonographic findings in confirmed cases of Beckwith-Wiedemann syndrome. Prenat Diagn 2015; 35: 394-399.

36 Romanelli V, Belinch A, Benito-sanz S et al: CDKN1C (p57 Kip2) analysis in BeckwithWiedemann syndrome (BWS) patients: genotype-phenotype correlations. Novel Mutat Polymorphisms 2010; 152A: 1390-1397.

37 Wollmann HA, Kirchner T, Enders H, Preece MA, Ranke MB: Growth and symptoms in Silver-Russell syndrome: review on the basis of 386 patients. Eur J Pediatr 1995; 154: 958-968.

38 Wakeling EL, Amero SA, Alders M et al: Epigenotype-phenotype correlations in SilverRussell syndrome. J Med Genet 2010; 47: 760-768.

39 Grønskov K, Poole RL, Hahnemann JMD et al: Deletions and rearrangements of the H19/IGF2 enhancer region in patients with Silver-Russell syndrome and growth retardation. J Med Genet 2011; 48: 308-311.

40 Azzi S, Blaise A, Steunou V et al: Complex tissue-specific epigenotypes in Russell-Silver syndrome associated with 11p15 ICR1 hypomethylation. Hum Mutat 2014; 35: 1211-1220.

41 Xiang Y, Zhang J, Li Q et al: DNA methylome profiling of maternal peripheral blood and placentas reveal potential fetal DNA markers for non-invasive prenatal testing. Mol Hum Reprod 2014; 20: 875-884.

42 Wong Al, Lo YM: Noninvasive fetal genomic, methylomic, and transcriptomic analyses using maternal plasma and clinical implications. Trends Mol Med 2015; 21: 98-108.

43 Garin I, Mantovani G, Aguirre U et al: European guidance for the molecular diagnosis of pseudohypoparathyroidism not caused by point genetic variants at GNAS: an EQA study. Eur J Hum Genet 2015; 23: 438-444.

44 Paganini L, Carlessi N, Fontana L et al: Beckwith-Wiedemann syndrome prenatal diagnosis by methylation analysis in chorionic villi. Epigenetics 2015; 10: 643-649. 